\title{
Relation of Some Known Functions in terms of Generalized Meijer G-Functions
}

\author{
Syed Ali Haider Shah, ${ }^{1}$ Shahid Mubeen $\mathbb{D}^{1},{ }^{1}$ Gauhar Rahman $\mathbb{D D}^{2}$ and Jihad Younis $\mathbb{D D}^{3}$ \\ ${ }^{1}$ Department of Mathematics, University of Sargodha, Sargodha, Pakistan \\ ${ }^{2}$ Department of Mathematics and Statistics, Hazara University, Mansehra, Pakistan \\ ${ }^{3}$ Department of Mathematics, Aden University, Khormaksar, P. O. Box 6014, Aden, Yemen
}

Correspondence should be addressed to Jihad Younis; jihadalsaqqaf@gmail.com

Received 11 September 2021; Accepted 18 October 2021; Published 3 November 2021

Academic Editor: Xiaolong Qin

Copyright ( 92021 Syed Ali Haider Shah et al. This is an open access article distributed under the Creative Commons Attribution License, which permits unrestricted use, distribution, and reproduction in any medium, provided the original work is properly cited.

\begin{abstract}
The aim of this paper is to prove some identities in the form of generalized Meijer $G$-function. We prove the relation of some known functions such as exponential functions, sine and cosine functions, product of exponential and trigonometric functions, product of exponential and hyperbolic functions, binomial expansion, logarithmic function, and sine integral, with the generalized Meijer $G$-function. We also prove the product of modified Bessel function of first and second kind in the form of generalized Meijer $G$-function and solve an integral involving the product of modified Bessel functions.
\end{abstract}

\section{Introduction}

The elementary functions such as trigonometric functions, exponential functions, logarithmic functions, hypergeometric functions, Bessel functions, Mittag-Leffler functions, and even binomial expansion are all special functions, which are widely applicable in many fields, especially in the field of sciences. The hypergeometric functions were firstly defined in series form by [1], also many properties were presented. Due to the wide range of applications, these hypergeometric functions have gained attention of the researchers. Almost all the elementary functions can be expressed in the form of hypergeometric functions.

The exponential functions, sine and cosine functions, and binomial expansion in the form of hypergeometric functions are given in $[2,3]$ as, respectively,

$$
\begin{aligned}
e^{\alpha} & =\sum_{v=0}^{\infty} \frac{\alpha^{v}}{v !}={ }_{0} F_{0}(-;-; \alpha), \\
\sin \alpha & =\sum_{v=0}^{\infty} \frac{(-1)^{v} \alpha^{2 v+1}}{v !(3 / 2)_{v} 2^{2 v}}={ }_{0} F_{1}\left(-; \frac{3}{2} ; \frac{-\alpha^{2}}{4}\right),
\end{aligned}
$$

$$
\cos \alpha=\sum_{v=0}^{\infty} \frac{(-1)^{v} \alpha^{2 v}}{v !(1 / 2)_{v} 2^{2 v}}={ }_{0} F_{1}\left(-; \frac{1}{2} ; \frac{-\alpha^{2}}{4}\right),
$$

where $\mathfrak{R}(\alpha)>0$ and

$$
(1-\alpha)^{-a}=\sum_{v=0}^{\infty} \frac{(a)_{v} \alpha^{v}}{v !}={ }_{1} F_{0}(a ;-; \alpha)
$$

The Bessel function is defined $[4,5]$ as

$$
\mathbf{j}_{1}(\alpha)=\sum_{v=0}^{\infty} \frac{(-1)^{v} \alpha^{2 v+1}}{v ! \Gamma(1+v+1) 2^{2 \mathrm{v}+1}} .
$$

Zhu et al. [6] estimated some weighted Simpson-like type integral inequalities, used them in some estimation type results to obtain the first-order differentiable functions, and applied them in some known special functions, modified Bessel function, $q$-digamma function, etc. Sarivastava et al. [7] worked on Mittag-Leffler type functions and estimated the Faber polynomial coefficient of biclose-to-convex functions connected with the Borel distribution of the Mittag-Leffler type. They considered the Fekete-Szegö type inequalities for biclose- 
to-convex function and also presented several results and related consequences. The researchers [8-10] also worked on inequalities involving special functions and proved various applications.

The concept of $k$-symbol was introduced by Diaz $[11,12]$. The $k$-theory gave boost to the field of special functions. The researchers [13-15] started to work on this particular $k$-symbol and proved many properties and identities.

Diaz defined Pochhammer $k$-symbol, gamma $k$-function, and beta $k$-function, for $k>0$, respectively, as

$$
\begin{aligned}
(l)_{m, k} & =(l)(l+k)(l+2 k) \cdots(l+(m-1) k), \quad m \geq 1, k>0, \\
\Gamma_{k}(l) & =\lim _{m \longrightarrow \infty} \frac{m ! k^{m}(m k)^{(l / k)-1}}{(l)_{m, k}}, \quad k>0, l \in \mathbb{C} \backslash k Z^{-}, \\
\beta_{k}(l, \omega) & =\frac{1}{k} \int_{0}^{1} x^{(l / k)-1}(1-x)^{(\omega / k)-1} \mathrm{~d} x, \quad \Re(l) \\
& >0, \mathfrak{R}(\omega)>0 .
\end{aligned}
$$

The integral form of gamma $k$-function is given as

$$
\Gamma_{k}(l)=\int_{0}^{\infty} x^{(l / k)-1} e^{\left(-x^{k} / k\right)} \mathrm{d} x, \quad \mathfrak{R}(l)>0, k>0 .
$$

Mubeen and Rehman [16] gave the $k$-form of some elementary functions.

The Meijer $G$-functions which are considered to be the general functions are the particular special functions which have gained the attention of many researchers [17-24]. Many special functions can be obtained by considering the specific variation of parameters of Meijer $G$-function. Most of the special functions are $G$-functions or can be expressed in the form of product of $G$-functions with elementary functions [25-29].

Meijer $G$-function is defined [30] as

$$
G_{p, q}^{m, n}\left[\begin{array}{c}
e_{p} \\
f_{q}
\end{array} \mid z\right]=\frac{1}{2 \pi i} \oint_{L} \frac{\Gamma\left(f_{1, m}-s\right) \Gamma\left(1-e_{1, n}+s\right)}{\Gamma\left(e_{n+1, p}-s\right) \Gamma\left(1-f_{m+1, q}+s\right)} z^{s} \mathrm{~d} s,
$$

where $\Gamma(\cdot)$ is well known gamma function and $0 \leq m \leq q, 0 \leq n \leq p, z \neq 0$.

The definitions of Meijer $G$-function in the form of hypergeometric function are given [31], respectively, as

$$
\begin{aligned}
G_{p, q}^{m, n}\left[\begin{array}{c}
e_{p} \\
f_{q}
\end{array} \mid z\right]= & \sum_{i=1}^{m} \frac{\prod_{h=1}^{m} \Gamma\left(f_{h}-f_{i}\right)^{*} \prod_{h=1}^{n} \Gamma\left(1-e_{h}+f_{i}\right) z^{f_{i}}}{\prod_{h=m+1}^{q} \Gamma\left(1-f_{h}+f_{i}\right) \prod_{h=n+1}^{p} \Gamma\left(e_{h}-f_{i}\right)} \\
& \times{ }_{p} F_{q-1}\left[\begin{array}{l}
1-e_{p}+f_{i} \\
\left(1-f_{q}+f_{i}\right)^{*}
\end{array}\right.
\end{aligned}
$$

for $p<q$ or $p=q,|z|<1$ and

$$
\begin{aligned}
G_{p, q}^{m, n}\left[\begin{array}{c}
e_{p} \\
f_{q}
\end{array} \mid z\right]= & \sum_{i=1}^{m} \frac{\prod_{h=1}^{n} \Gamma\left(e_{i}-e_{h}\right)^{*} \prod_{h=1}^{m} \Gamma\left(1-e_{i}+f_{h}\right) z^{e_{i}-1}}{\prod_{h=n+1}^{p} \Gamma\left(1-e_{i}+e_{h}\right) \prod_{h=m+1}^{q} \Gamma\left(e_{i}-f_{h}\right)} \\
& \times{ }_{q} F_{p-1}\left[\begin{array}{l}
1-e_{i}+f_{q} \\
\left(1-e_{i}+e_{p}\right)^{*}
\end{array} \mid(-1)^{q-m-n} z^{-1}\right],
\end{aligned}
$$

for $p>q$ or $p=q$ and $|z|>1$.

The relation of Meijer $G$-functions with elementary functions as exponential, logarithmic, cosine, and Bessel functions is given by Santosh [32] as

$$
\begin{aligned}
& e^{t}=G_{1,0}^{0,1}\left[\begin{array}{l|l}
- & -t \\
0 & -
\end{array},\right. \\
& \ln |1+t|=G_{2,2}^{1,2}\left[\begin{array}{l}
1,1 \\
1,0
\end{array} \mid t\right], \\
& \cos t=\sqrt{\pi} G_{0,2}^{1,0}\left[\begin{array}{c|c}
- & t^{2} \\
0, \frac{1}{2} & \frac{4}{4}
\end{array}\right] \text {, }
\end{aligned}
$$

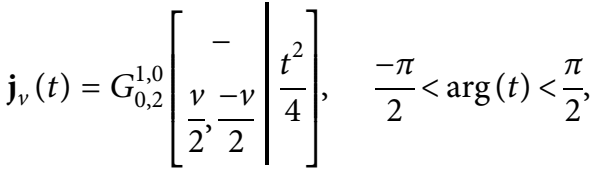

$$
\begin{aligned}
& \left.M(t)=\frac{1}{2} G_{2,2}^{1,2}\left[\begin{array}{c|c}
\frac{1}{2}, & \frac{1}{2} \\
0,0
\end{array}\right]-t\right],
\end{aligned}
$$

where $\mathbf{j}_{v}(t)$ and $M(t)$ are the Bessel and modified Bessel functions, respectively.

Roach [33] gave the relation of hypergeometric functions with Meijer $G$-function as

$$
F(e ; f ; z)=\frac{\Gamma(f)}{\Gamma(e)} G(1-e ; ; 0 ; 1-f ; \log (-z)),
$$

and proved some results related to Meijer $G$-function. Dyda et al. [34] worked on fractional Laplace operators and Meijer $G$-functions and discussed some transformation properties of Meijer $G$-function given as

$$
\begin{aligned}
& t^{d} G_{p, q}^{m, n}\left[\begin{array}{l}
e \\
f
\end{array} \mid t\right]=G_{p, q}^{m, n}\left[\begin{array}{l}
e+d \mid \\
f+d
\end{array}\right], \\
& G_{p, q}^{m, n}\left[\begin{array}{l}
e \\
f
\end{array} \mid t^{-1}\right]=G_{p, q}^{m, n}\left[\begin{array}{c}
1-e \\
1-f
\end{array} \mid t\right], \\
& G_{p, q}^{m, n}\left[\begin{array}{l}
d, e^{\prime} \\
f^{\prime}, d
\end{array} \mid t\right]=G_{p-1, q-1}^{m, n-1}\left[\begin{array}{l}
e^{\prime} \\
f^{\prime}
\end{array} \mid t\right], \\
& G_{p, q}^{m, n}\left[\begin{array}{l}
e^{\prime}, d \\
d, f^{\prime}
\end{array} \mid t\right]=G_{p-1, q-1}^{m-1, n}\left[\begin{array}{l}
e^{\prime} \\
f^{\prime}
\end{array} \mid t\right],
\end{aligned}
$$

where $e^{\prime}=\left(e_{1}, e_{2}, \ldots, e_{p-1}\right)$ and $f^{\prime}=\left(f_{1}, f_{2}, \ldots, f_{p-1}\right)$. Pishkoo and Darus [35] gave series representations of three 
basic univalent $G$-functions by using Mellin-Barnes type contour integral representation.

In our current findings, we first define the generalized form of Meijer $G$-function in the integral and hypergeometric forms and obtain some known special functions such as the Bessel function, exponential function, sine function, cosine function, sine and cosine hyperbolic functions, product of exponential and trigonometric functions, product of exponential and hyperbolic functions, binomial expansions, and logarithmic function by using the definitions of generalized Meijer $G$-functions for different choice of parameters. We define the generalized form of modified Bessel functions of the first and second kind and prove results for the product of modified Bessel functions in the form of generalized Meijer $G$-function.

\section{Generalized Meijer G-Functions and Their Relation with Some Known Functions}

In this section, we define the generalized form of (7) and (8) in the form of hypergeometric $k$-function and (6) in integral form for $k>0$. The relations of some known functions with generalized Meijer $G$-function are also considered.

Definition 1. For $p<q$ or $p=q$ and $|z|<1$, the generalized Meijer $G$-function can be defined as

$$
\begin{aligned}
G_{k, p, q}^{m, n}\left[\begin{array}{c}
e_{p} \\
f_{q}
\end{array} \mid z\right]= & \sum_{i=1}^{m} \frac{\prod_{h=1}^{m} \Gamma_{k}\left(f_{h}-f_{i}\right)^{*} \prod_{h=1}^{n} \Gamma_{k}\left(k-e_{h}+f_{i}\right) z^{f_{i} / k}}{\prod_{h=m+1}^{q} \Gamma_{k}\left(k-f_{h}+f_{i}\right) \prod_{h=n+1}^{p} \Gamma_{k}\left(e_{h}-f_{i}\right)} \\
& \times{ }_{p} F_{q-1, k}\left[\begin{array}{c}
k-e_{p}+f_{i} \\
\left(k-f_{q}+f_{i}\right)^{*}
\end{array} \mid(-1)^{p-m-n} z\right] .
\end{aligned}
$$

Definition 2. For $p>q$ or $p=q$ and $|z|>1$, the generalized

Meijer $G$-function can be defined as

$$
\begin{aligned}
G_{k, p, q}^{m, n}\left[\begin{array}{c}
e_{p} \\
f_{q}
\end{array} \mid z\right]= & \sum_{i=1}^{m} \frac{\prod_{h=1}^{n} \Gamma_{k}\left(e_{i}-e_{h}\right)^{*} \prod_{h=1}^{m} \Gamma_{k}\left(k-e_{i}+f_{h}\right) z^{\left(e_{i} / k\right)-1}}{\prod_{h=n+1}^{p} \Gamma_{k}\left(k-e_{i}+e_{h}\right) \prod_{h=m+1}^{q} \Gamma_{k}\left(e_{i}-f_{h}\right)} \\
& \times{ }_{q} F_{p-1, k}\left[\begin{array}{c}
k-e_{i}+f_{q} \\
\left(k-e_{i}+e_{p}\right)^{*}
\end{array} \mid(-1)^{q-m-n} z^{-1}\right] .
\end{aligned}
$$

Definition 3. The integral form of generalized Meijer $G$-function for $k>0$ is given as

$$
G_{k, p, q}^{m, n}\left[\begin{array}{c}
e_{p} \\
f_{q}
\end{array} \mid z\right]=\frac{1}{2 \pi i} \oint_{L} \frac{\Gamma_{k}\left(f_{1, m}-s\right) \Gamma_{k}\left(k-e_{1, n}+s\right)}{\Gamma_{k}\left(e_{n+1, p}-s\right) \Gamma_{k}\left(k-f_{m+1, q}+s\right)} z^{s / k} \mathrm{~d} s,
$$

where $\Gamma_{k}(\cdot)$ is the well-known gamma $k$-function and $0 \leq m \leq q, 0 \leq n \leq p, z \neq 0$.

Now, we prove some known special functions by considering different choices of parameters of generalized Meijer $G$-functions defined in (12) and (13), for $k>0$. Different identities are proved as follows.
Proposition 1. Let $f_{1}=(\alpha+b) / 2, \quad f_{2}=(-\alpha+b) / 2$, $z=\left(x^{2} / 4\right), p=0, q=2, m=1, n=0$, then (12) gives the generalized Meijer G-function in the form of Bessel function as

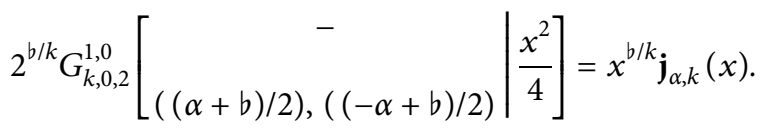

Proof. By choosing $f_{1}=(\alpha+b) / 2, \quad f_{2}=(-\alpha+b) / 2$, $z=\left(x^{2} / 4\right), p=0, q=2, m=1, n=0$ in (12), we have

$$
\begin{aligned}
& G_{k, 0,2}^{1,0}\left[\begin{array}{c|c}
- & \frac{x^{2}}{4}
\end{array}\right]=\frac{\left(x^{2} / 4\right)^{(\alpha+b) / 2 k}}{\Gamma_{k}(k+((\alpha-b) / 2)+t((\alpha+b) / 2))}{ }_{0} F_{1, k}\left[\begin{array}{c}
- \\
k+\alpha
\end{array} \mid \frac{-x^{2}}{4}\right] \\
& =\frac{x^{b / k}}{2^{b / k}} \mathbf{j}_{\alpha, k}(x),
\end{aligned}
$$


which gives the desired result.

Proposition 2. Let $e_{1}=0, e_{2}=k / 2, f_{1}=0, f_{2}=k / 2$, $z=x, p=2, q=2, m=1, n=1$, then (12) gives

$$
\left.\frac{\pi}{k} G_{k, 2,2}^{1,1}\left[\begin{array}{c}
0, \frac{k}{2} \\
0, \frac{k}{2}
\end{array}\right] x\right]=(1+k x)^{-1} \text {. }
$$

Proof. By choosing $e_{1}=0, e_{2}=k / 2, f_{1}=0, f_{2}=k / 2, z=$ $x, p=2, q=2, m=1, n=1$ in (12), we have

$$
\begin{aligned}
G_{k, 2,2}^{1,1}\left[\begin{array}{c}
0, \frac{k}{2} \\
0, \frac{k}{2} \mid x
\end{array}\right] & =\frac{\Gamma_{k}(k)}{\Gamma_{k}(k-(k / 2)) \Gamma_{k}(k / 2)}{ }_{2} F_{1, k}\left[\begin{array}{c}
k, \frac{k}{2} \mid \\
\frac{k}{2} \mid
\end{array}\right] \\
& =\frac{1}{\Gamma_{k}(k / 2) \Gamma_{k}(k / 2)} \sum_{n=0}^{\infty} \frac{(k)_{n, k}(-1)^{n} x^{n}}{n !} .
\end{aligned}
$$

Since $\Gamma_{k}(k / 2)=\sqrt{\pi / k}$, so

$$
G_{k, 2,2}^{1,1}\left[\begin{array}{c}
0, \frac{k}{2} \\
0, \frac{k}{2}
\end{array}\right] x=\frac{k}{\pi}(1+k x)^{-1} .
$$

Proposition 3. Let $e_{1}=k-\rho, f_{1}=0, z=x, p=1, q=1$, $m=1, n=1$, then (12) gives the relation of Meijer $G$-function with binomial series as

$$
\frac{1}{\Gamma_{k}(\rho)} G_{k, 1,1}^{1,1}\left[\begin{array}{c|c}
k-\rho & x \\
0 & x
\end{array}\right]=(1+k x)^{-\rho / k} .
$$

Proof. By choosing $e_{1}=k-\rho, f_{1}=0, z=x, p=1, q=1$, $m=1, n=1$ in (12), we have

$$
\begin{aligned}
G_{k, 1,1}^{1,1}\left[\begin{array}{c|c}
k-\rho & \\
0 & x
\end{array}\right] & =\Gamma_{k}(\rho)_{1} F_{0, k}\left[\begin{array}{l}
\rho \\
-
\end{array}\right] \\
& =\Gamma_{k}(\rho) \sum_{n=0}^{\infty} \frac{(\rho)_{n, k}(-1)^{n} x^{n}}{n !} \\
& =\Gamma_{k}(\rho)(1+k x)^{-\rho / k} .
\end{aligned}
$$

Proposition 4. Let $e_{1}=\beta, f_{1}=0, z=x, p=1, q=1, m=$ $1, n=0$ in (12), then the generalized Meijer G-function can be expressed in series form as

$$
G_{k, 1,1}^{1,0}\left[\begin{array}{l|l}
\beta & \\
0 & x
\end{array}\right]=\frac{1}{\Gamma_{k}(\beta)} \sum_{n=0}^{\infty} \frac{(k-\beta)_{n, k} x^{n}}{n !} .
$$

Proof. By choosing $e_{1}=\beta, f_{1}=0, z=x, p=1, q=1, m=$ $1, n=0$ in (12), we have

$$
\begin{aligned}
G_{k, 1,1}^{1,0}\left[\begin{array}{l}
\beta \\
0
\end{array} \mid x\right] & =\frac{1}{\Gamma_{k}(\beta)}{ }_{1} F_{0, k}\left[\begin{array}{c}
k-\beta \mid x \\
-
\end{array}\right] \\
& =\frac{1}{\Gamma_{k}(\beta)} \sum_{n=0}^{\infty} \frac{(k-\beta)_{n, k} x^{n}}{n !} .
\end{aligned}
$$

Corollary 1. By taking same choices as in the above case in (13), we can obtain

$$
\Gamma_{k}(\beta) G_{k, 1,1}^{1,0}\left[\begin{array}{l}
\beta \\
0
\end{array}\right]=\sum_{n=0}^{\infty} \frac{(k-\beta)_{n, k}}{x^{n} n !} .
$$

Proposition 5. Let $f_{1}=(k / 2), f_{2}=0, z=x, p=0, q=2$, $m=1, n=0$, then (12) gives the relation of generalized Meijer G-function with sine function as

$$
\sqrt{\pi} G_{k, 0,2}^{1,0}\left[\frac{-}{\frac{k}{2}, 0} \mid x\right]=\sin 2\left(\sqrt{\frac{x}{k}}\right) .
$$

Proof. By choosing $f_{1}=(k / 2), f_{2}=0, z=x, p=0, q=2$, $m=1, n=0$ in (12), we have

$$
\begin{aligned}
G_{k, 0,2}^{1,0}\left[\frac{k}{2}, 0 \mid x\right] & =\frac{x^{1 / 2}}{\Gamma_{k}(3 k / 2)}{ }_{0} F_{1, k}\left[\frac{3 k}{2} \mid-x\right] \\
& =\frac{2 x^{1 / 2}}{\sqrt{k \pi}} \sum_{n=0}^{\infty} \frac{(-x)^{n}}{(3 k / 2)_{n, k} n !} \\
& =\frac{1}{\sqrt{\pi}} \sin 2\left(\sqrt{\frac{x}{k}}\right) .
\end{aligned}
$$

Proposition 6. Let $f_{1}=0, f_{2}=(k / 2), z=x, p=0, q=2$, $m=1, n=0$, then (12) gives the relation of generalized Meijer G-function with cosine function as

$$
\sqrt{\pi} G_{k, 0,2}^{1,0}\left[\begin{array}{c|c}
- & k \\
0, \frac{k}{2} & x
\end{array}=\sqrt{k} \cos 2\left(\sqrt{\frac{x}{k}}\right) .\right.
$$

Proof. By choosing $f_{1}=0, f_{2}=(k / 2), z=x, p=0, q=2$, $m=1, n=0$ in (12), we have 


$$
\begin{aligned}
G_{k, 0,2}^{1,0}\left[0, \frac{k}{2} \mid x\right] & =\frac{1}{\Gamma_{k}(k / 2)}{ }_{0} F_{1, k}\left[\begin{array}{l}
- \\
\frac{k}{2}
\end{array}\right] \\
& =\frac{\sqrt{k}}{\sqrt{\pi}} \sum_{n=0}^{\infty} \frac{(-x)^{n}}{(k / 2)_{n, k} n !} \\
& =\sqrt{\frac{k}{\pi}} \cos 2\left(\sqrt{\frac{x}{k}}\right) .
\end{aligned}
$$

Proposition 7. Let $e_{1}=k, f_{1}=0, z=x k, p=1, q=0, m=$ $0, n=1$ in (12), then we have

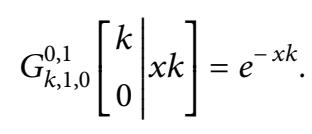

Proof. By choosing $e_{1}=k, f_{1}=0, z=x k, p=1, q=0, m=$ $0, n=1$ in (12), we have

$$
\begin{aligned}
G_{k, 1,0}^{0,1}\left[\begin{array}{l}
k \\
0
\end{array} \mid x k\right] & ={ }_{0} F_{0, k}\left[\begin{array}{l}
- \\
-
\end{array}\right] \\
& =\sum_{n=0}^{\infty} \frac{(-x k)^{n}}{n !}=e^{-x k} .
\end{aligned}
$$

Corollary 2. By taking the same choice of parameters as in the previous proposition and $z=x$, then (13) gives

$$
G_{k, 1,0}^{0,1}\left[\begin{array}{c|c}
k & x \\
0 & x
\end{array}\right]=e^{-1 / x} \text {. }
$$

Proof. By choosing $e_{1}=k, f_{1}=0, z=x, p=1, q=0, m=$ $0, n=1$ in (13), we obtain

$$
\begin{aligned}
& G_{k, 1,0}^{0,1}\left[\begin{array}{l|l}
k & x \\
0 & x
\end{array}\right]={ }_{0} F_{0, k}\left[\begin{array}{l}
-\mid-x^{-1} \\
-\mid
\end{array}\right] \\
& =\sum_{n=0}^{\infty} \frac{(-1)^{n}}{(x)^{n} n !} \\
& =e^{\frac{-1}{x}} .
\end{aligned}
$$

Proposition 8. Let $e_{1}=k, f_{1}=k, f_{2}=0, z=x, p=1, q=$ $2, m=1, n=1$, then (12) gives the relation of generalized Meijer G-function with the product of exponential and sine hyperbolic function as

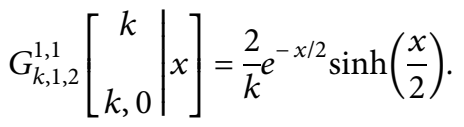

Proof. By choosing $e_{1}=k, f_{1}=k, f_{2}=0, z=x, p=1, q=$ $2, m=1, n=1$ in (12), we have

$$
\begin{aligned}
G_{k, 1,2}^{1,1}\left[\begin{array}{c|c}
k & \\
k, 0 & x
\end{array}\right] & =\frac{x}{\Gamma_{k}(2 k)}{ }_{1} F_{1, k}\left[\begin{array}{c}
k \\
2 k
\end{array}\right] \\
& =\frac{x}{k} \sum_{n=0}^{\infty} \frac{(k)_{n, k}(-x)^{n}}{(2 k)_{n, k} n !} \\
& =\frac{2}{k} e^{-x / 2} \sinh \left(\frac{x}{2}\right) .
\end{aligned}
$$

Corollary 3. For $e_{1}=0, e_{2}=k, f_{1}=0, z=x, p=2, q=1$, $m=1, n=1$, (13) gives

$$
G_{k, 2,1}^{1,1}\left[\begin{array}{c|c}
0, k & x \\
0 & x
\end{array}\right]=\frac{2}{k} e^{-1 / 2 x} \sinh \left(\frac{1}{2 x}\right) .
$$

Proof. By choosing $e_{1}=0, e_{2}=k, f_{1}=0, z=x, p=2, q=$ $1, m=1, n=1$ in (13), we can obtain

$$
\begin{aligned}
G_{k, 2,1}^{1,1}\left[\begin{array}{c}
0, k \\
0
\end{array} \mid x\right] & =\frac{x^{-1}}{\Gamma_{k}(2 k)}{ }_{1} F_{1, k}\left[\begin{array}{c}
k \\
2 k
\end{array} \mid-x^{-1}\right] \\
& =\frac{1}{k} \sum_{n=0}^{\infty} \frac{(k)_{n, k}(1 / x)^{n+1}(-1)^{n}}{(2 k)_{n, k} n !} \\
& =\frac{2}{k} e^{-1 / 2 x} \sinh \left(\frac{1}{2 x}\right) .
\end{aligned}
$$

Proposition 9. Let $e_{1}=(k / 2), e_{2}=(3 k / 2), z=x, p=2, q=$ $0, m=0, n=1$ in (13), then

$$
\left.\sqrt{\pi} G_{k, 2,0}^{0,1}\left[\begin{array}{c}
\frac{k}{2}, \frac{3 k}{2} \\
-
\end{array}\right] x\right]=\sin 2(k x)^{-1 / 2} .
$$

Proof. By choosing $e_{1}=(k / 2), \quad e_{2}=(3 k / 2)$, $z=x, p=2, q=0, m=0, n=1$ in (13), we obtain

$$
\begin{aligned}
\left.G_{k, 2,0}^{0,1}\left[\begin{array}{c}
\frac{k}{2}, \frac{3 k}{2} \\
-
\end{array}\right] x\right] & \left.=\frac{x^{-1 / 2}}{\Gamma_{k}(3 k / 2)}{ }_{0} F_{1, k}\left[\begin{array}{c}
- \\
\frac{3 k}{2}
\end{array}\right]-x^{-1}\right] \\
& =\frac{1}{\Gamma_{k}(3 k / 2)} \sum_{n=0}^{\infty} \frac{(-1)^{n}}{(3 k / 2)_{n, k}(x)^{n+(1 / 2)} n !} \\
& =\frac{1}{\sqrt{\pi}} \sin 2(k x)^{-1 / 2} .
\end{aligned}
$$

Proposition 10. Let $e_{1}=0, e_{2}=k, e_{3}=(k / 2), f_{1}=0$, $f_{2}=0, f_{3}=(k / 2), \quad z=x, p=3, q=3, m=2, n=1$, then 
(13) gives the relation of generalized Meijer G-function with logarithmic function as

$$
k \pi G_{k, 3,3}^{2,1}\left[\begin{array}{c}
0, k, \frac{k}{2} \mid \\
0,0, \frac{k}{2} \mid
\end{array}\right]=\ln \left|1-\frac{k}{x}\right| .
$$

Proof. By choosing $e_{1}=0, e_{2}=k, e_{3}=(k / 2), f_{1}=0$, $f_{2}=0, f_{3}=(k / 2), z=x, p=3, q=3, m=2, n=1$ in (13), we have

$$
\begin{aligned}
& G_{k, 3,3}^{2,1}\left[\begin{array}{c}
0, k, \frac{k}{2} \\
0,0, \frac{k}{2}
\end{array} \mid x\right]=\frac{x^{-1}}{\Gamma_{k}(-k / 2) \Gamma_{k}(3 k / 2) \Gamma_{k}(2 k)}{ }_{3} F_{2, k} \\
& {\left[\begin{array}{c|c}
k, k, \frac{3 k}{2} & \frac{1}{x} \\
2 k, \frac{3 k}{2} &
\end{array}\right]} \\
& =\frac{-2}{\pi} \sum_{n=0}^{\infty} \frac{(k)_{n, k}}{(2 k)_{n, k}(x)^{n+1} n !} \\
& =\frac{1}{k \pi} \ln \left|1-\frac{k}{x}\right| \text {. }
\end{aligned}
$$

Proposition 11. Let $e_{1}=(k / 2), e_{2}=k, e_{3}=k, f_{1}=0 z=$ $x, p=3, q=1, m=1, n=1$ in (13), then we have

$$
G_{k, 3,1}^{1,1}\left[\begin{array}{c|c}
\frac{k}{2}, k, k & x \\
0 &
\end{array}\right]=\frac{2}{k \sqrt{\pi}} \operatorname{Si}\left(\frac{2}{\sqrt{k x}}\right),
$$

where $S i$ is the sine integral.

Proof. By choosing $e_{1}=(k / 2), e_{2}=k, e_{3}=k, f_{1}=0 z=$ $x, p=3, q=1, m=1, n=1$ in (13), we have

$$
\begin{aligned}
\left.G_{k, 3,1}^{1,1}\left[\begin{array}{c}
\frac{k}{2}, k, k \\
0
\end{array}\right] x\right] & \left.=\frac{x^{-1 / 2} \Gamma_{k}(k / 2)}{\Gamma_{k}(3 k / 2) \Gamma_{k}(3 k / 2)}{ }_{1} F_{2, k}\left[\begin{array}{c}
\frac{k}{2} \\
\frac{3 k}{2}, \frac{3 k}{2}
\end{array}\right]-\frac{1}{x}\right] \\
& =\frac{4}{k \sqrt{k \pi}} \sum_{n=0}^{\infty} \frac{(k / 2)_{n, k}(-1)^{n}}{(3 k / 2)_{n, k}(3 k / 2)_{n, k} x^{n+1 / 2} n !} \\
& =\frac{2}{k \sqrt{\pi}} \operatorname{Si}\left(\frac{2}{\sqrt{k x}}\right) .
\end{aligned}
$$

\section{Product of Modified Bessel Functions in terms of Generalized Meijer G-Function and Their Relations}

In this section, we first define the modified Bessel function of the first and second kind in the form of generalized Meijer $G$-function and then prove the relation of the product of modified Bessel functions with the generalized Meijer $G$-function by taking the specific choice of parameter of the Bessel functions. At the end, we solve an integral involving the product of modified Bessel functions in the integrand.

For complex argument $x$, the modified Bessel functions of the first and second kind are defined, respectively, as

$$
\begin{aligned}
& l_{v, k}(x)=\iota^{-v} G_{k, 0,2}^{1,0}\left[\begin{array}{c|c}
- & \frac{-x^{2}}{2}, \frac{-v k}{2} \\
\frac{4}{2}
\end{array}\right], \\
& M_{v, k}(x)=\frac{1}{2} G_{k, 0,2}^{2,0}\left[\begin{array}{c|c}
\frac{v k}{2}, \frac{-v k}{2} & \frac{x^{2}}{4}
\end{array}\right],
\end{aligned}
$$

where $v$ is an integer.

Theorem 1. Let $v=3$ in (43) and (44), then the product of modified Bessel functions in terms of generalized Meijer G-function is given as

$$
l_{3, k}(x) M_{3, k}(x)=\frac{\sqrt{k}}{2 \sqrt{\pi}} G_{k, 1,3}^{2,1}\left[\begin{array}{c}
\frac{k}{2} \\
3 k, 0,-3 k
\end{array} \mid x^{2}\right] .
$$

Proof. We first consider the left hand side as

$$
\begin{aligned}
l_{3, k}(x)= & \iota^{-3} G_{k, 0,2}^{1,0}\left[\frac{3 k}{2}, \frac{-3 k}{2} \mid \frac{-x^{2}}{4}\right] \\
= & \frac{-1}{\imath \Gamma_{k}(4 k)} \sum_{j=0}^{\infty} \frac{(-1)^{2 j+(3 / 2)} x^{2 j+3}}{(4 k)_{j, k} j ! 2^{2 j+3}} \\
= & \frac{1}{8 \Gamma_{k}(4 k)} \sum_{j=0}^{\infty} \frac{x^{2 j+3}}{(4 k)_{j, k} j ! 2^{2 j}}, \\
M_{3, k}(x)= & \frac{1}{2} G_{k, 0,2}^{2,0}\left[\frac{3 k}{2}, \frac{-3 k}{2} \mid \frac{x^{2}}{4}\right] \\
= & \frac{1}{2}\left[\Gamma_{k}(-3 k) \sum_{j=0}^{\infty} \frac{x^{2 j+3}}{(4 k)_{j, k} n ! 2^{2 j+3}}\right. \\
& \left.+\Gamma_{k}(3 k) \sum_{j=0}^{\infty} \frac{x^{2 j-3}}{(-2 k)_{j, k} j ! 2^{2 j-3}}\right] .
\end{aligned}
$$


So, from (46) and (47), we have

$$
\begin{aligned}
l_{3, k}(x) M_{3, k}(x)= & {\left[\frac{1}{8 \Gamma_{k}(4 k)} \sum_{j=0}^{\infty} \frac{x^{2 j+3}}{(4 k)_{j, k} j ! 2^{2 j}}\right] } \\
& \cdot\left[\frac { 1 } { 2 } \left[\Gamma_{k}(-3 k) \sum_{j=0}^{\infty} \frac{x^{2 j+3}}{(4 k)_{j, k} j ! 2^{2 j+3}}\right.\right. \\
& \left.\left.+\Gamma_{k}(3 k) \sum_{j=0}^{\infty} \frac{x^{2 j-3}}{(-2 k)_{j, k} j ! 2^{2 j-3}}\right]\right] .
\end{aligned}
$$

By working on (48), we obtain

$$
\begin{aligned}
l_{3, k}(x) M_{3, k}(x)= & \frac{\Gamma_{k}(-3 k)}{128 \Gamma_{k}(4 k)} \sum_{j=0}^{\infty} \frac{(7 k / 2)_{j, k} x^{2 j+6}}{(4 k)_{j, k}(7 k)_{j, k} j !} \\
& +\frac{\Gamma_{k}(3 k)}{2 \Gamma_{k}(4 k)} \sum_{j=0}^{\infty} \frac{(k / 2)_{j, k} x^{2 j}}{(-2 k)_{j, k}(4 k)_{j, k} j !} .
\end{aligned}
$$

Now, by considering the right hand side and applying (12), we have

$$
\begin{aligned}
G_{k, 1,3}^{2,1}\left[\begin{array}{c|c}
\frac{k}{2} \\
3 k, 0,-3 k
\end{array} \mid x^{2}\right]= & \frac{\Gamma_{k}(-3 k) \Gamma_{k}(7 k / 2)}{\Gamma_{k}(7 k)} \sum_{j=0}^{\infty} \frac{(7 k / 2)_{j, k} x^{2 j+6}}{(4 k)_{j, k}(7 k)_{j, k} j !} \\
& +\frac{\Gamma_{k}(3 k) \Gamma_{k}(k / 2)}{\Gamma_{k}(4 k)} \sum_{j=0}^{\infty} \frac{(k / 2)_{j, k} x^{2 j}}{(-2 k)_{j, k}(4 k)_{j, k} j !} .
\end{aligned}
$$

From (49) and (50), we obtain the required result.

Theorem 2. Let $u$ be the variable of integration, $k>0$, and $\sqrt{u z}$ be the argument of the modified Bessel functions of the first and second kind, then we have

$$
\left.\int_{0}^{\infty} e^{u^{k} / k} G_{k, 1,3}^{2,1}\left[\begin{array}{c}
\frac{k}{2} \\
3 k, 0,-3 k
\end{array}\right] u z\right] \mathrm{d} u=G_{k, 2,3}^{2,2}\left[\begin{array}{c|c}
\frac{k}{2}, 0 \\
3 k, 0,-3 k
\end{array}\right] z
$$

Proof. From Theorem 3.1, we can write

$$
\begin{aligned}
l_{3, k}(\sqrt{u z}) M_{3, k}(\sqrt{u z})= & \left.\frac{\sqrt{k}}{2 \sqrt{\pi}} G_{k, 1,3}^{2,1}\left[\begin{array}{c|c}
\frac{k}{2} \\
3 k, 0,-3 k
\end{array}\right] u z\right] \\
= & \frac{\Gamma_{k}(-3 k) \Gamma_{k}(7 k / 2)}{\Gamma_{k}(7 k)} \sum_{j=0}^{\infty} \frac{(7 k / 2)_{j, k}(u z)^{j+3}}{(4 k)_{j, k}(7 k)_{j, k} j !} \\
& +\frac{\Gamma_{k}(3 k) \Gamma_{k}(k / 2)}{\Gamma_{k}(4 k)} \sum_{j=0}^{\infty} \frac{(k / 2)_{j, k}(u z)^{j}}{(-2 k)_{j, k}(4 k)_{j, k} j !} .
\end{aligned}
$$

Now, multiplying by $e^{u^{k} / k}$ and taking integral, we have

$$
\begin{aligned}
\int_{0}^{\infty} e^{u^{k} / k} l_{3, k}(\sqrt{u z}) M_{3, k}(\sqrt{u z}) \mathrm{d} u= & \frac{\Gamma_{k}(-3 k) \Gamma_{k}(7 k / 2)}{\Gamma_{k}(7 k)} \sum_{j=0}^{\infty} \frac{(7 k / 2)_{j, k}(z)^{j+3}}{(4 k)_{j, k}(7 k)_{j, k} j !} \int_{0}^{\infty}(u)^{j+3} \frac{e^{u^{k}}}{k} \mathrm{~d} u \\
& +\frac{\Gamma_{k}(3 k) \Gamma_{k}(k / 2)}{\Gamma_{k}(4 k)} \sum_{j=0}^{\infty} \frac{(k / 2)_{j, k}(z)^{j}}{(-2 k)_{j, k}(4 k)_{j, k} j !} \int_{0}^{\infty}(u)^{j} e^{u^{k} / k} \mathrm{~d} u \\
= & \frac{\Gamma_{k}(-3 k) \Gamma_{k}(7 k / 2) \Gamma_{k}(4 k)}{\Gamma_{k}(7 k)} \sum_{j=0}^{\infty} \frac{(7 k / 2)_{j, k}(4 k)_{j, k}(z)^{j+3}}{(4 k)_{j, k}(7 k)_{j, k} j !} \\
& +\frac{\Gamma_{k}(3 k) \Gamma_{k}(k / 2) \Gamma_{k}(k)}{\Gamma_{k}(4 k)} \sum_{j=0}^{\infty} \frac{(k / 2)_{j, k}(k)_{j, k}(z)^{j}}{(-2 k)_{j, k}(4 k)_{j, k} j !} \\
\int_{0}^{\infty} e^{u^{k} / k} G_{k, 1,3}^{2,1}\left[\begin{array}{l}
k \\
2
\end{array}\right] \mathrm{d} u= & \frac{\Gamma_{k}(-3 k) \Gamma_{k}(7 k / 2) \Gamma_{k}(4 k)}{\Gamma_{k}(7 k)} \sum_{j=0}^{\infty} \frac{(7 k / 2)_{j, k}(4 k)_{j, k}(z)^{j+3}}{(4 k)_{j, k}(7 k)_{j, k} j !} \\
& +\frac{\Gamma_{k}(3 k) \Gamma_{k}(k / 2) \Gamma_{k}(k)}{\Gamma_{k}(4 k)} \sum_{j=0}^{\infty} \frac{(k / 2)_{j, k}(k)_{j, k}(z)^{j}}{(-2 k)_{j, k}(4 k)_{j, k} j !} .
\end{aligned}
$$


Also, by using (12) on the right hand side, we have

$$
\begin{aligned}
\left.G_{k, 2,3}^{2,2}\left[\begin{array}{c}
\frac{k}{2}, 0 \\
3 k, 0,-3 k
\end{array}\right] z\right]= & \frac{\Gamma_{k}(-3 k) \Gamma_{k}(7 k / 2) \Gamma_{k}(4 k)}{\Gamma_{k}(7 k)} \sum_{j=0}^{\infty} \frac{(7 k / 2)_{j, k}(4 k)_{j, k}(z)^{j+3}}{(4 k)_{j, k}(7 k)_{j, k} j !} \\
& +\frac{\Gamma_{k}(3 k) \Gamma_{k}(k / 2) \Gamma_{k}(k)}{\Gamma_{k}(4 k)} \sum_{j=0}^{\infty} \frac{(k / 2)_{j, k}(k)_{j, k}(z)^{j}}{(-2 k)_{j, k}(4 k)_{j, k} j !}
\end{aligned}
$$

Hence, from (53) and (54), we obtained the desired result.

\section{Conclusions}

In this research work, we gave some functions as sine, cosine, exponential, logarithmic functions, etc., in terms of the generalized form of Meijer $G$-function. We proved the theorems, which gave the relation of the product of Bessel functions of the first and second kind with the generalized Meijer G-function, and solved the integral involving modified Bessel functions as an integrand, respectively.

\section{Data Availability}

The data used to support the findings of this study are available from the corresponding author upon request.

\section{Conflicts of Interest}

The authors declare that they have no conflicts of interest.

\section{Authors' Contributions}

All the authors contributed equally and they read and approved the final manuscript for publication.

\section{References}

[1] C. F. Gauss, "Disquisitiones generales circa seriem infinitam," Comm. soc. reg. sci. Gott. rec.vol. 3, no. 2, pp. 123-162, 1812.

[2] E. D. Rainville, Special Functions, The Macmillan Co. Inc., New York, NY, USA, 1960.

[3] I. S. Gradshteyn and I. M. Ryzhik, Tables of Integrals, Series, and Products, Academic Press, Amsterdam, Boston, Sydney, Tokyo, Seventh edition, 2007.

[4] A. Gray, E. Gray, G. Mathews, and E. Meissel, A Treatise on Bessel Functions and Their Applications to Physics, Macmillan \& Company, New York, NY, USA, 1895.

[5] D. N. Tumakov, "The faster methods for computing Bessel functions of the first kind of an integer order with applications to graphic processes," Labchevskii Journal of Mathematics, vol. 40, pp. 1752-1738, 2019.

[6] T. Zhu, P. Wang, and T. Du, "Some estimates ON the weighted simpson-like type integral inequalities and their applications," Journal of Nonlinear Functional Analysis, vol. 2020, Article ID 17, 2020.
[7] H. M. Srivastava, G. Murugusundaramoorthy, and S. M. ElDeeb, "Faber polynomial coefficient estmates of bi-close-toconvex functions connected with the Borel distribution of the Mittag-Leffler type," Journal of Nonlinear and Variational Analysis, vol. 5, pp. 103-118, 2021.

[8] S.-Y. Tan, T.-R. Huang, and Y.-M. Chu, "Functional inequalities for Gaussian hypergeometric function and generalized elliptic integral of the first kind," Mathematica Slovaca, vol. 71, no. 3, pp. 667-682, 2021.

[9] M. K. Wang, Y. M. Chu, and Y. P. Jiang, "Ramanujan's cubic transformation inequalities for zero balanced hypergeometric functions," Rocky Mountain Journal of Mathematics, vol. 46, pp. 679-691, 2016.

[10] S.-L. Qiu, X.-Y. Ma, and Y.-M. Chu, "Sharp Landen transformation inequalities for hypergeometric functions, with applications," Journal of Mathematical Analysis and Applications, vol. 474, no. 2, pp. 1306-1337, 2019.

[11] R. Díaz and C. Teruel, " $q, k$-generalized gamma and beta functions," Journal of Nonlinear Mathematical Physics, vol. 12, no. 1, pp. 118-134, 2005.

[12] R. Diaz and E. Pariguan, "On hypergeometric functions and pochhammer $k$-symbol," Divulgaciones mathematicas, vol. 15, pp. 179-192, 2007.

[13] M. Mansour, "Determining the $k$-generalized gamma function by functional equations," International Journal of Contemporary Mathematical Sciences, vol. 4, pp. 1037-1042, 2009.

[14] S. Mubeen and G. M. Habibullah, " $k$-fractional integrals and application," International Journal of Contemporary Mathematical Sciences, vol. 7, pp. 89-94, 2012.

[15] C. G. Kokologiannaki, "Properties and inequalities of generalized $k$-gamma, beta and zeta functions," International Journal of Contemp. Math. Sci.vol. 5, pp. 653-660, 2010.

[16] S. Mubeen and A. Rehman, " $(n, k)$-factorials," Journal of Inequalities and Special Functions, vol. 5, no. 3, 2014.

[17] Y. L. Luke, The Special Functions and Their Approximations, Vol. 1, Academic Press, Cambridge, MA, USA, 1969.

[18] L. J. Slater, Generalized Hypergeometric Functions, Cambridge University Press, Cambridge, MA, USA, 1996.

[19] V. Adamchik, "The evaluation of integrals of Bessel functions via G-function identities," Journal of Computational and Applied Mathematics, vol. 64, no. 3, pp. 283-290, 1995.

[20] A. M. Mathai, R. K. Saxena, and H. J. Haubold, The HFunction Theory and Applications, Springer-Verlag, New York Dordrecht Heidelberg London, 2010.

[21] C. S. Meijer, "Expansion theorems for the G-function. V," Indagationes Mathematicae, vol. 56, pp. 349-357, 1953.

[22] N. E. Norlund, "Hypergeometric functions," Acta Mathematica, vol. 94, no. 1, pp. 289-349, 1955. 
[23] A. P. Prudnikov, Y. A. Brychkov, and O. I. Marichev, "Integrals and series," More Special Functions, Vol. 3, Gordon and Breach Science Publications, New York, NY, USA, 1990.

[24] A. Pishkoo, "A G-function form of radial states in carbon and hydrogen atoms: absorption or emission of radiation," Advanced Studies in Theoretical Physics, vol. 9, no. 3, pp. 145-154, 2015.

[25] R. Beals and J. Szmigielski, "Meijer G-functions: a gentle introduction," Notices of the American Mathematical Society, vol. 60, no. 6, pp. 866-872, 2013.

[26] A. P. Prudnikov, Y. A. Brychkov, and O. I. Marichev, "Integrals and series," Direct Laplace Transform, Vol. 4, Gordon and Breach Science Publishers, New York, NY, USA, 1992.

[27] A. P. Prudnikov, Y. A. Brychkov, and O. I. Marichev, "Integrals and series," Inverse Laplace Transform, Vol. 5, Gordon and Breach Science Publishers, New York, NY, USA, 1992.

[28] A. Erdélyi, W. Magnus, F. Oberhettinger, and F. G. Tricomi, Tables of Integral Transforms, vol. I, McGraw-Hill Book Co. Inc., New York, Toronto and London, 1954, Bateman Manuscript Project.

[29] A. Erdélyi, W. Magnus, F. Oberhettinger, and F. G. Tricomi, Higher Transcendental Function, Vol. III, McGraw -Hill Book Company, New York, Toronto and London, 1955.

[30] A. A. Kilbas, R. K. Saxena, M. Saigo, and J. J. Trujillo, “The generalized hypergeometric function as the Meijer G-function," Analysis, vol. 36, no. 1, pp. 1-14, 2016.

[31] J. L. Fields, "The asymptotic expansion of the meijer G-function," Mathematics of Computation, vol. 26, no. 119, pp. 757-765, 1972.

[32] S. Kumar, "Exact evaluations of some Meijer G-functions and probability of all eigenvalues real for the product of two Gaussian matrices," Journal of Physics A: Mathematical and Theoretical, vol. 48, no. 44, Article ID 445206, 2015.

[33] K. Roach, "Meijer G-function representations," in Proceedings of the 1997 International Symposium on Symbolic and Algebraic Computation, pp. 205-211, Kihei, HI, USA, July 1997.

[34] B. Dyda, A. Kuznetsov, and M. Kwaśnicki, "Fractional laplace operator and meijer G-function," Constructive Approximation, vol. 45, no. 3, pp. 427-448, 2017.

[35] A. Pishkoo and M. Darus, "Relationship between path and series representations for the three basic univalent $G$-functions," Journal of Advances in Mathematics, vol. 6, no. 1, 2014. 